\title{
Long-Term Complete Remission of Refractory Primary Cutaneous Anaplastic Large T Cell Lymphoma Treated with Brentuximab Vedotin: A Case Report
}

\author{
Loseto $\mathbf{G}^{1}$, Ciavarella $\mathbf{S}^{1 *}$, Scattone $\mathbf{A}^{2}$, Calabrese $\mathbf{A}^{3}$, Quinto $\mathbf{A M}^{1}$ and Guarini $\mathbf{A}^{1}$ \\ ${ }^{1}$ Hematology Unit, IRCCS - Istituto Tumori "G. Paolo II", Bari, Italy \\ ${ }^{2}$ Pathology Unit, IRCCS - Istituto Tumori "G. Paolo II", Bari, Italy \\ ${ }^{3}$ Radiology Unit, IRCCS - Istituto Tumori "G. Paolo II", Bari, Italy
}

\begin{abstract}
Based on early results from a single phase III trial, Brentuximab Vedotin (BV), a drug-conjugated anti-CD30 monoclonal antibody, is changing the treatment strategy, especially for relapsed/refractory cutaneous anaplastic large cell lymphomas (CALCL) patients with limited therapeutic options. However, despite high response rates registered in this setting, follow-up data about duration of response and long-term toxicity profile are still lacking. Here, we report a case of refractory, advanced stage CALCL in a 78-year old patient with poor prognosis, which showed a dramatic, long-lasting response even to reduced doses of BV as salvage treatment.
\end{abstract}

Keywords: Anti-CD30 therapy; Cutaneous anaplastic $\mathrm{T}$ cell lymphoma

\section{Introduction}

Although most primary cutaneous Anaplastic Large Cell Lymphomas (pc-ALCL) display an indolent course and common spontaneous remission, elderly patients with advanced stage (T2-3) disease, characterized by extensive involvement of limb or head and neck regions, have poor prognosis and currently represent a challenge for clinicians [1]. The recognition of high CD30 antigen expression in pc-ALCL has recently prompted the design of prospective clinical trials aimed at evaluating the therapeutic efficacy of Brentuximab Vedotin (BV) (Adcetris), an anti-CD30 monoclonal antibody conjugated with an antimitotic microtubule inhibitor (monomethyl auristatin E), in this subset of patients [2]. Here, we report a case of refractory, stage T3 pcALCL in a 78-year old patient suffering of extensive multifocal skin involvement, which showed a complete and long-term response to BV even when used at reduced dosage as salvage treatment.

\section{Case}

A 78-year-old man with previous history of type 2 diabetes mellitus treated with oral hypoglycemic and ischemic cardiomyopathy came to our attention in January 2012 in concomitance with skin nodular lesions compatible with advanced-staged refractory cutaneous ALCL (first diagnosis established in 2010; stage T3bN0M0). Previous treatments consisted in six cycles of CHOP chemotherapy (cyclophosphamide, doxorubicin, vincristine and reduced doses of prednisone) leading to partial remission assessed in December 2010. Between January 2011 and June 2011, he underwent unsuccessful treatment with Bexarotene (Bex) (150 mg daily) in combination with Methotrexate (Mtx) (12 mg intramuscularly twice weekly). Then, the patient was treated with Bex (150 mg daily) associated with gemcitabine $\left(800 \mathrm{mg} / \mathrm{m}^{2}\right.$ on day 1,8 and 15 of 28-day cycle), suspended after a total of 3 cycles in October 2011 due to the lack of substantial objective response.

At time of our observation, a CT/PET scan showed extensive cutaneous involvement indicating, in absence of bone marrow disease infiltration, a T3bN0M0 disease stage. Histological examination of surgical excisional skin biopsy confirmed the diagnosis of CD30positive ALCL (Figures $1 \mathrm{~A}$ and $1 \mathrm{~B}$ ) expressing CD4, CD3, LCA and EMA, negative for CD20, CD15, CD10, BCL6, ALK and with $90 \%$
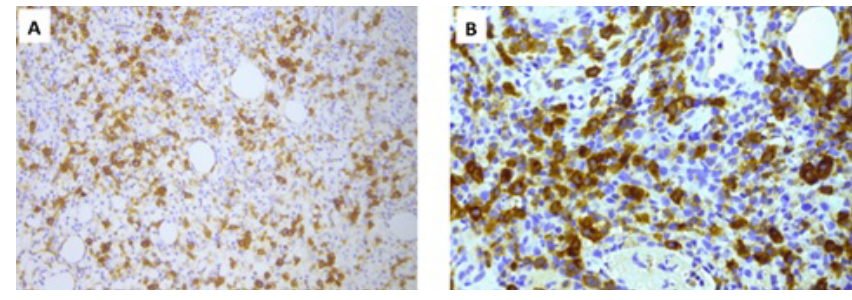

Figure 1: Representative 20X (A) and 40X (B) optical microscopy images showing CD30-positive (brown immunohistochemical signal) large cells infiltrating the histological tumor specimen.

Ki67. The disease was clinically evident as papules and nodules at skin of trunk, right shoulder and forearm left groin and foot. The patient was treated with Romidepsin (at dosage of $14 \mathrm{mg} / \mathrm{m}^{2}$ on day 1,8 and 15 of 28 -day cycle, subsequently reduced to $10 \mathrm{mg} / \mathrm{m}^{2}$ for a total of 8 cycles due to hematological toxicity), showing initial partial response followed by a clear cutaneous progression. Thus, the patient was addressed to 3 monthly cycles of Bendamustine ( $90 \mathrm{mg} / \mathrm{m}^{2}$ on day $1-2$ of 28 -day cycle), stopped after 3 cycles due to evident therapeutic failure and cutaneous disease progression characterized by rapidly growing nodules with focal ulcerative lesions (Figures 2A and 2D). Given the advanced patient age, poor performance status (increasing fatigue and weakness) and the initial development of renal insufficiency (serum creatinine equal to $2.2 \mathrm{mg} / \mathrm{dl}$ associated with progressive diffuse edema), in November 2013, he was addressed to an off-label dose-reduced schedule of BV monotherapy $(1.2 \mathrm{mg} / \mathrm{kg}$ on day 1 of 21 -day cycle). After the first cycle, the mentioned lesions showed striking reduction (Figure 2B)

*Corresponding author: Sabino Ciavarella, MD, PhD Hematology Unit, IRCCS - Istituto Tumori “G. Paolo II” Viale O. Flacco, 6570125 Bari, Italy, Tel: 390805555416; E-mail: s.ciavarella@oncologico.bari.it

Received June 07, 2017; Accepted June 26, 2017; Published June 27, 2017

Citation: Loseto G, Ciavarella S, Scattone A, Calabrese A, Quinto AM, et al. (2017) Long-Term Complete Remission of Refractory Primary Cutaneous Anaplastic Large T Cell Lymphoma Treated with Brentuximab Vedotin: A Case Report. J Cancer Sci Ther 9: 503-504. doi: 10.4172/1948-5956.1000467

Copyright: ( 2017 Loseto G, et al. This is an open-access article distributed under the terms of the Creative Commons Attribution License, which permits unrestricted use, distribution, and reproduction in any medium, provided the original author and source are credited. 
Citation: Loseto G, Ciavarella S, Scattone A, Calabrese A, Quinto AM, et al. (2017) Long-Term Complete Remission of Refractory Primary Cutaneous Anaplastic Large T Cell Lymphoma Treated with Brentuximab Vedotin: A Case Report. J Cancer Sci Ther 9: 503-504. doi: 10.4172/19485956.1000467

until achieving a complete clinical disappearance even at the end of the second cycle. In particular, major nodules at right groin and foot skin underwent rapid shrinking and, after about 50 days of treatment, appeared as minute hyperemic scary lesions (Figures 2C and 2E).

On this basis, a total of 12 cycles were completed and no significant toxic effects were observed. The last disease evaluation was performed in December 2016. The patient was asymptomatic and the disease showed persistent complete remission even at month 36 after BV treatment.

\section{Discussion}

$\mathrm{BV}$ is an anti-CD30 monoclonal antibody conjugated with the microtubule polymerization inhibitor monomethyl auristatin E, already approved for the treatment of refractory Hodgkin lymphoma and systemic ALCL [2]. To date, only few case reports [3-6], two phase II studies $[7,8]$, and early results from the ongoing phase III "ALCANZA" trial [9] reported impressive and rapid clinical response to BV, which commonly occur even within the first two cycles of therapy. In particular, the "ALCANZA" study included 31 refractory pcALCL and 97 mycosis fungoides (MF) patients randomized to receive BV $1.8 \mathrm{mg} /$ $\mathrm{kg}$ once every 3 weeks or physician's choice (Mtx $5 \mathrm{mg}$ to $50 \mathrm{mg}$ or Bex $300 \mathrm{mg} / \mathrm{m}^{2}$ ), showing overall response and complete response rates of $75 \%$ and $31 \%$ respectively for the pcALCL group. Notably, for all study patients, the median progression free survival was of 16.7 at a median follow-up of 17.5 months [9]. Data from longer follow-up are thus warranted to provide crucial information about the duration of response to $\mathrm{BV}$ in these patients.

Data from our case are in line with the above results, as the clinical efficacy of BV was evident even after the second cycle of therapy in a patient in which previous lines of treatments (CHOP, Bex, Mtx, Gemcitabine, Bendamustine and Romidepsin) have had been unsuccessful. Most importantly, BV was highly effective even if used at dose as low as $1.2 \mathrm{mg} / \mathrm{kg}$ and its toxicity profile was negligible. This is
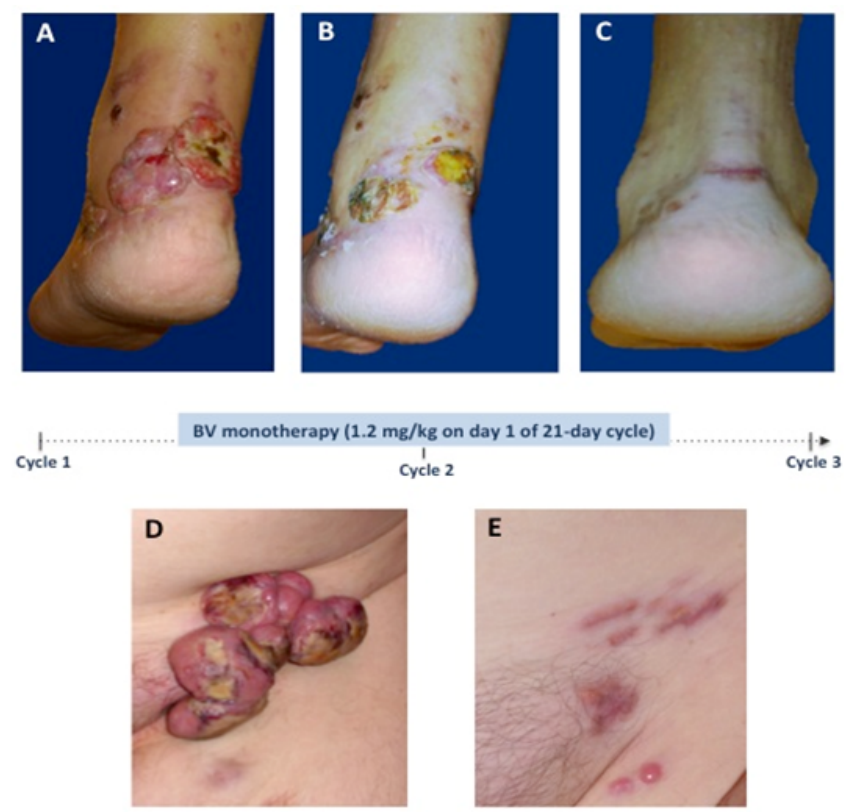

Figure 2: Pictures showing $\mathrm{CALCL}$ skin localizations as papules and ulcerating nodules at left hell and groin regions before starting Brentuximab Vedotin (BV) $(A, D)$, after the first cycle of treatment $(B)$ and at the end of the second cycle (C, E), when they appeared as tiny, hyperemic superficial scars. of particular advantage when treating elderly patients bearing multiple co-morbidities.

\section{Conclusion}

Finally, based on our long-term follow-up, we are able to report a long-lasting complete remission (36 months), unexpectedly obtained in a patient with many poor prognostic factors including older age, advanced stage with multiple localizations and extensive limb involvement.

In the light of results from In the light of early results from the ongoing clinical trial "ALCANZA" and looking for more updated follow-up information, we provide demonstration that BV produces not only dramatic but also durable responses in pcALCL, and carries minimal risks of adverse toxic effects, thus being of great advantage for treating elderly, heavily pretreated patients with no alternative options of therapy

\section{Conflict of interest}

The Authors have no conflict of interest to declare.

\section{References}

1. Zinzani PL, Bonthapally V, Huebner D, Lutes R, Chi A, et al. (2016) Panoptic clinical review of the current and future treatment of relapsed/refractory T-cel lymphomas: Cutaneous T-cell lymphomas. Crit Rev Oncol Hematol 99: 228240.

2. Kumar A, Younes A (2014) Role of CD30 targeting in malignant lymphoma. Curr Treat Options Oncol 15: 210-225

3. Desai A, Telang GH, Olszewski AJ (2013) Remission of primary cutaneous anaplastic large cell lymphoma after a brief course of brentuximab vedotin. Ann Hematol 92: 567-568.

4. Mody K, Wallace JS, Stearns DM, Bowers G, Lacy SR, et al. (2013) CD30 Positive cutaneous T-Cell Lymphoma and response to Brentuximab Vedotin: 2 illustrative cases. Clin Lymphoma Myeloma Leuk 13: 319-323.

5. Kaffenberger BH, Winardi, FK, Frederickson J, Porcu P, Wong HK (2013) Periocular cutaneous anaplastic large cell lymphoma clearance with Brentuximab Vedotin. J Clin Aesthet Dermatol 6: 29-31.

6. Patsinakidis N, Kreuter A, Moritz RKC, Stücker M, Altmeyer P, et al. (2015) Complete remission of refractory, ulcerated, primary cutaneous CD30 + anaplastic large cell lymphoma following Brentuximab Vedotin therapy. Acta Derm Venereol 95: 233-234.

7. Duvic M, Tetzlaff MT, Gangar P, Clos AL, Sui D, et al. (2015) results of a phase II trial of Brentuximab Vedotin for CD30+ cutaneous T-cell lymphoma and lymphomatoid papulosis. J Clin Oncol 33: 3759-3765.

8. Kim YH, Tavallaee M, Sundram U, Salva KA, Wood GS, et al.(2015) Phase II investigator-initiated study of Brentuximab Vedotin in mycosis fungoides and sézary syndrome with variable CD30 expression level: A multi-institution collaborative project. J Clin Oncol 33: 3750-3758.

9. Kim YH, Whittaker S, Horwitz SM, Duvic M, Dummer R, et al. (2016) Brentuximab Vedotin demonstrates significantly superior clinical outcomes in patients with CD30-expressing cutaneous T cell lymphoma versus physician's choice (Methotrexate or Bexarotene): The phase 3 Alcanza study. ASH meeting, San Diego December 3 2016, Abstract 182 